Because this wire-wire gridless touch performs satisfactorily, no further research and development on touch-sensitive surface is being planned at LRDC. Development efforts are being directed towards touch-sensitive surface applications and the design and construction of new student terminals incorporating touch-sensitive screens.

The total cost of the units constructed is somewhat difficult to estimate since they were constructed as prototypes in our own shops. The cost estimates used internally for the units are:

Wire-Wire Grid Touch

Materials $\$ 50$
Labor and overhead

TOTAL

$\$ 650$

Wire-Wire Gridless Touch

Materials

$\$ 150$

Labor and overhead

TOTAL

$\$ 800$

REFERENCES

BOOKER, C. A., DOW, B. R., \& LAM BRIGHT, J, E. Graphic input tablets for programmed instruction. Technical Report 6, 1966, Learning Research and Development Center, University of Pittsburgh, Pittsburgh, Pennsylvania.

GLASER, R., RAMAGE, W., \& LIPSON, J. The interface between student and subject matter. Technical Report 5, 1964,
Learning Research and Development Center, University of Pittsburgh, Pittsburgh, Pennsylvania.

JUDD, W. A. The development of an on-line laboratory for CAI and behavioral research (1964-1968). Technical Report Series, 1969, Learning Research and Development Center, University of Pittsburgh, Pittsburgh, Pennsylvania.

\section{NOTES}

1. Polacoat flexible Lenscreen Type LS60FM is available from Polacoat, Inc., Blue Ash, Ohio 45242. The price is $\$ 2.50 / \mathrm{sq} \mathrm{ft}$.

2. This terminal is described in more detail in a technical report entitled, "An advanced touch sensitive display terminal," currently in preparation.

3. NESA-coated glass is available from PPG Ind ustries, 632 Fort Duquesne Boulevard, Pittsburgh, Pennsylvania 15222 . The price, for small quantities, is approximately $\$ 30 / \mathrm{sq} \mathrm{ft}$ for the $1 / 4-\mathrm{in}$. thickness.

\title{
Two-voice music programming system: The PDP-9 as an automatic electronic organ
}

\author{
PHILLIP L. EMERSON, ELIZABETH CAMUS, and CHRIS RICHARDS \\ Cleveland State University, Cleveland, Ohio 44115
}

A small computer was programmed to translate two-part musical scores into tables of numbers which were then used in the execution of the music through an amplifier and loudspeaker. The objective was to create sets of tape recordings to be used in psychophysical testing of the discriminabilities and preferabilities of music played on different pitch scales. The close tolerances in the timing of events in such stimulus materials demand special programming techniques and hardware components. The working system is described, and recommendations are made concerning the main features of an improved system.

A small computer has many uses in a psychological laboratory, and it may seem curious that valuable computer time would be used to play music. Our purpose is to prepare stimulus materials for research on the discriminabilities and preferabilities of different musical scales. A particularly interesting question for such research is whether or not people with and without musical training can tell the difference when the same musical composition is played on two particular scales: the just-intonational scale and the equal-tempered scale. For definitions and discussions of these and other scales, we refer to the classic and still highly relevant source, Helmholtz's Tonemphindungen, and especially the translation by $A$. J. Ellis (1930), with copious annotations and appendices. Under conditions where these two scales are discriminable, the question becomes: which is preferable?

In the experimental design for the investigation of these questions, a single stimulus presentation needs to be a sequence of several notes at least, and the esthetic question really becomes interesting only if the sequences are "pieces" of music self-contained in the sense of a song, a verse, or a movement from a larger composition. Initially, we intend to hold the sequence length and the nominal pitch pattern constant for the most part, while varying, as the main independent variable, the actual pitches slightly about those of the "standard" (just intonational) scale. This entails something like the experimental determination of a psychometric function in the space of 11 dimensions, since it is possible to vary 11 of the 12 pitches independently relative to the others. In many simple pieces of music, however, the dimensionality would reduce to 6 , corresponding to the seven natural notes of the major scale. Moreover, these 6 variables are each restricted essentially to a small region in the positive half range of real numbers, since they can be mapped to the six intervals between the successive pitches on the 7-point scale, Nevertheless, the investigation would be extremely ambitious if carried out by the classical individual psychophysical methods associated with signal detection theory, for example. Therefore, the initial experiments are planned as group experiments in which data are collapsed over individuals as well as trials. From those results, perhaps it will be clear that the detuning of any given pitch in the scale has essentially the same amount of effect as the detuning of any other, and that the effects are additive or combinable in some other way. If so, it should then be possible to define a new single independent variable accounting for the effects of the original 11 or 6 . If not, then the particular interaction patterns would be of great interest.

Thus, the experiments would be conducted somewhat in the manner of group audiometry, but with some practice exposure to the sequence played on the standard scale, and with the experimental trials being of the two-alternative forced-choice type. But problems emerge in the control of extraneous variables and even of the primary variable of interest. Ordinary musical instruments are not well suited for changing from one musical scale to another. Even with instruments where such changes are possible, it probably is a rare musician who can adhere precisely to a given musical scale and shift at will from one to another. A computer can be programmed to do these things on command, and it has other obvious advantages for this kind of research. These advantages stem from the exact reproducibility of a sequence of events, and the controllability of changes from one sequence to another. THE PDP-9 PROGRAMS

The system of programs for the 
performance of two-part musical arrangements consists of three programs which we call simply Programs 1, 2, and 3 .

Program No. 1 is written in SNOBOL, and it translates the music from a modified musical notation to times and half periods of the pitches of the notes to be played. It is fairly simple and contains the tables wherein the pitches of the musical scales are specified. This program takes its input from a file on DECtape, previously created by means of the PDr-9 software system program editor. It writes its output in another file on DECtape which becomes the input file for Program No. 2.

Program No. 2 does most of the numerical computing, and it was written partly in FORTRAN and partly in assembly language. It takes lines from its input file, each representing a note to be played, and creates for each one a lookup table of times in microseconds between the events that will be output to produce the acoustical end result, It writes these successive lookup tables into an output file which becomes the input file for Program No. 3.

Program No. 3 executes the performance of the music translated by Programs 1 and 2. It reads in and plays the notes provided to it in the file which is output from Program 2.

It would be possible to combine these programs if there were any significant advantage in doing so. But the modularity provided by three segments has proven useful for making small program changes and for changing parameter values, most of which are specified in Program 1, which can be edited and rerun quickly. It consists of about 100 SNOBOL statements which are interpreted directly by SNOBOL.

\section{THE OBJECTIVES, HARDW ARE CONSTRAINTS, AND THE SOLUTION}

We had some programs to play a single melodic voice. The sound was produced by connecting an amplifier and speaker to a single flip-flop bit of an output buffer similar to the relay buffer which is a standard option on the PDP-9. The programs produced tones by complementing the contents of the bit periodically at twice the frequency of the pitch of the desired note. The periodic switching of the flip-flop produces essentially a square wave, $f(t)$

$$
f(t)=\left\{\begin{array}{c}
1, k \pi<t \leqslant(k+1) \pi \\
-1,(k+1)<t \leqslant(k+2) \pi
\end{array}\right.
$$

for $\mathrm{k}=0, \pm 1, \pm 2, \ldots$ where the time unit is adjusted for convenience so that the half period is $\pi$ units.

The amplitude, $A_{j}$, of its $j^{\text {th }}$ harmonic is given by

$$
A_{j}=\sqrt{a_{j}^{2}+b_{j}^{2}}
$$

where

$a_{j}=\left\lfloor\rho_{0}^{2 \pi} f(t) \cos (j t) d t\right] /\left[\int_{0}^{2 \pi} \cos ^{2}(j t) d t \mid\right.$ and

$\mathbf{b}_{\mathrm{j}}=\left[\int_{0}^{2 \pi} \mathrm{f}(\mathrm{t}) \sin (\mathrm{jt}) \mathrm{dt}\right] /\left[\int_{0}^{2 \pi} \sin ^{2}(\mathrm{jt}) \mathrm{dt}\right]$.

From considerations of symmetry, it can be shown that $a_{j}=0$ for $j=1,2$, $\ldots$ and therefore that $A_{j}=b_{j}$. The necessary values of the other integrals can be looked up in a table or determined from familiar identities. It turns out that

$$
A_{j}=\left\{\begin{array}{l}
0, j \text { even } \\
4 / j \pi, j \text { odd }
\end{array}\right.
$$

This pattern is qualitatively similar to those typical of double reed woodwinds, having significant contributions from the odd harmonics and nothing from the even ones. Presumably, the higher harmonics were further attenuated by the low-pass filtering effects of the bass-selection network of the amplifier and by the mechanical properties of the speaker. The tonal quality did not seem objectionable.

Alternatively, the tones might be produced by such devices as binary d i g i tal-to-frequency or analogue-to-frequency converters, but such approaches open the door to the problems of maintaining the calibration of tuning of the external wave generators.

The PDP-9 was equipped with the standard 60-cycle clock, three DECtapes, a custom-designed buffered microsecond clock, and the binary output buffer (18 bits) mentioned above. The microsecond clock consists essentially of a $1-\mathrm{MHz}$ crystal and two 18-bit registers, a counting register and a buffer register. After the clock is started by the execution of a certain I/O instruction, it normally operates with the counter counting microseconds upward toward zero through the two's complement negative series. When it reaches zero, the device flag goes on, causing an interrupt if the program interrupt of the PDP-9 is enabled. Thereupon, the number in the buffer is transferred automatically to the counter, and another count up to zero is begun immediately without any missed pulses. Thus, a sequence of exactly timed intervals may be produced. They need not be equal, since the buffer may be reloaded with a new number from the PDP-9 accumulator. This is done between interrupts by the execution of a certain $1 / 0$ instruction. A design limitation of the clock is that a time less than about 50 microsec cannot be obtained. Another limitation is the time needed to service an interrupt. The latter is about the same as the former in most cases, so there is no point in redesigning the clock.

With the equipment described above, the objective was to write programs to perform translations from some convenient notation for entering two-part musical script on the Teletype and to execute these translations. The execution was to be accomplished by switching the states of two bits of the output buffer at the appropriate times. No amplitude variation was envisioned, since $D / A$ converters were not available, but they could be incorporated easily. The execution program (No. 3 ) was to be able to play notes already read in while concurrently reading in notes to be played next, so that the sequence length would be limited by the capacity of a DECtape rather than by core memory. The 60-cycle clock was to be used to time the length of each note. The microsecond clock would provide the time intervals for complementing the bits of the two channels at the appropriate times to produce the two periodic square waves.

The minimum of 50 microsec obtainable from the clock proved to be the most fundamental limitation. It was necessary to make approximations to the pitches to be mixed, adjusting one or both slightly to make the ratio a fairly simple rational fraction. Let $h_{1}$ and $h_{2}$ be the adjusted half periods of the two pitches to be mixed, and let $n$ be the greatest common integral divisor of $h_{1}$ and $h_{2}$. Then the half period of the mixture is $h_{1} h_{2} / n$. Assume $h_{1}<h_{2}$. Then the smallest nonzero interval that needs to be produced by the clock is the smaller of $d$ and $h_{1}-d$, where $d$ is the remainder of the integer division, $h_{2} / h_{1}$. The number of ordered interval $x$ bit-pattern combinations in one period of the mixture is $2\left[\left(h_{1}+h_{2}\right) / n\right]-2$, and the scheme was to precompute for each pitch combination in sequence, a table of this many numbers for quick indexed lookup after each clock interrupt during the actual acoustical execution. The idea was to minimize the clock-interrupt service time to maximize the upper limit of complexity of the mixture, while leaving enough spare time for the DECtapes to keep ahead of the acoustical execution in the delivery of fresh tables. To the same end, the 


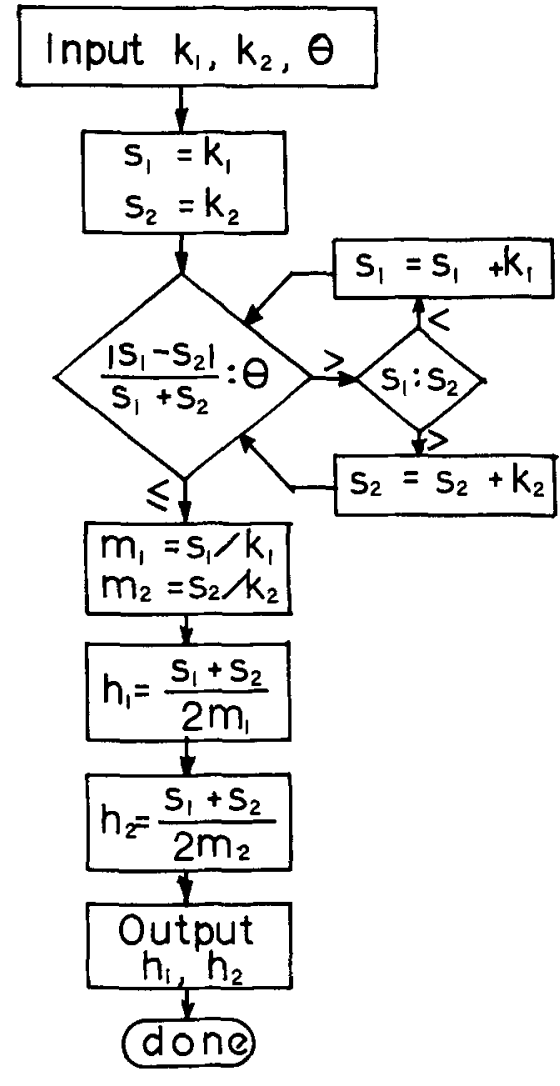

Fig. 1. Flow chart of the routine for computing the approximations, $h_{1}$ and $h_{2}$, to the half periods, $k_{1}$ and $k_{2}$, of the pitches to be mixed.

PDP-9 monitor system was regenerated with the microsecond clock skip-on-flag instruction at the head of the skip chain to give it the highest priority on simultaneous interrupts. Also, the tables to be read in by Program 3 during execution are written by Program 2 in dump mode to save a little processing time in input. Program 3 contains 16 buffers so that the DECtape can stay 15 notes ahead of execution normally, with this backlog being decreased temporarily in fast cacophonic passages.

Figure 1 illustrates the details of the routine for the rational approximation mentioned above. There, $k_{1}$ and $k_{2}$ are the half periods of the pitches to be mixed, as specified in the output of Program 1 in the form of integers where the unit of time is 1 microsec. The parameter $\theta$ is a threshold whose value is specified via the keyboard when Program 2 is run. The routine ends with approximations $h_{1}$ and $h_{2}$, such that $m_{1} h_{1}=m_{2} h_{2}$, where $m_{1}$ and $m_{2}$ are integers whose final values depend on the value of $\theta$. The division operations whence the values of $h_{1}$ and $h_{2}$ are obtained must be truncated or rounded, since $h_{1}$ and $h_{2}$ must finally be given in integral numbers of microseconds. Hence, when the table of intervals is formed on the basis of these values of $h_{1}$ and $h_{2}$, there may still be a discrepancy between $m_{1} h_{1}$ and $m_{2} h_{2}$. To avoid severe unwanted acoustical effects, the program distributes such a discrepancy symmetrically in the period of the mixture. Even so, there is occasionally a marked combination tone at the pitch corresponding to the period of the mixture.

Thus, the two main limitations of the present system, and apparently of almost any system attempting to do the same things with the same hardware, are (1) the minimum clock interval of 50 microsec and (2) the 1 -microsec resolution of the clock. The effect of the first limitation of the present system is that the pitches to be mixed must form a rather simple ratio, especially if they are very high (say two octaves above middle $C$ ). The effect of the second limitation is an occasional strong "difference" tone. These limitations have clear implications for certain additional hardware components.

\section{SOME IMPROVEMENTS POSSIBLE WITH ADDITIONAL}

HARDWARE COMPONENTS

The improvements considered here will exclude any that would mean giving up the main desirable properties designed into the system described above. These properties are (1) concurrent input and acoustical execution to provide uninterrupted execution of long sequences, (2) internal generation of waveforms to avoid retuning and recalibration of external wave generators, and (3) the modular parameterization in regard to tempo and the pitches of the musical scale.

One system having these desired properties without the limitations described above would consist of the basic PDP-9 or a similar small computer with DECtapes or an equivalent mass-storage device, plus two buffered microsecond clocks similar to the one described above and two double-buffered D/A converters. Each channel would then consist of a microsecond clock whose flag was gated directly to trigger its own respective $\mathrm{D} / \mathrm{A}$ converter to perform a conversion, i.e., to transfer the contents of the D/A buffer register to the conversion register. Such a system would also provide the capability of amplitude variation, but, more important, the direct link of each microsecond clock to its own D/A converter would avoid the necessity of lookup-tables of variable intervals, and the accuracy of the produced intervals would not be affected by small delays in the servicing of the clock interrupts. If this is not clear, consider the following sequence of events at the end of a clock count on one channel during the production of square waves of different frequencies on the two channels: (1) The microsecond-clock flag goes on, the clock begins timing again with the same interval, D/A conversion occurs, program interrupt occurs; (2) device flag is cleared, control is transferred to the service routine; (3) the service routine saves the accumulator, link, and return address; (4) it can reenable the interrupt here, since there is plenty of time before another interrupt from this channel will occur; e.g., about 250 microsec for three octaves above middle $\mathrm{C}$; (5) the service routine complements the contents of the D/A converter buffer; (6) it restores the accumulator and link; and (7) returns.

Total time for the execution of the above sequence of steps is about 40 microsec, and the sequence can be interrupted by the other channel or by other devices without ill effects. In fact, such a system could be expanded to three or four channels such as these, which would be out of the question for the system presently in operation. The microsecond clocks of the different channels could all draw their pulses from the same crystal.

REFERENCE

HELMHOLTZ, H. L. F, von. (Translated by A. J. Ellis) Sensations of tone. (5th ed.) New York: Longmans, Green, 1930. 